CLINICAL STUDY

\title{
Mitotane has a strong and a durable inducing effect on CYP3A4 activity
}

Nielka P van Erp, Henk-Jan Guchelaar, Bart A Ploeger ${ }^{1}$, Johannes A Romijn ${ }^{2}$, Jan den Hartigh and Hans Gelderblom ${ }^{3}$

Department of Clinical Pharmacy and Toxicology, Leiden University Medical Center, PO Box 9600, Postal zone LO-P, 2300 RC Leiden, The Netherlands, ${ }^{1}$ LAPEP Consultants BV, Leiden, The Netherlands and Departments of ${ }^{2}$ Endocrinology and Metabolic Diseases and ${ }^{3}$ Clinical Oncology, Leiden University Medical Center, 2300 RC Leiden, The Netherlands

(Correspondence should be addressed to N P van Erp; Email: p.h.van_erp@lumc.nl)

\begin{abstract}
Objective: The effects of mitotane on the pharmacokinetics (PK) of co-administered drugs are mostly unknown. The aim of the present study was to describe the effects of mitotane on the PK of the phenotypic probe midazolam and of the tyrosine kinase inhibitor sunitinib.

Design: A serendipitous observation was made in two of nine patients who volunteered in a sunitinib pharmacokinetic study. Both patients were diagnosed with adrenocortical carcinoma (ACC) and were exposed to mitotane. The sunitinib PK study was designed to determine the relationship between CYP3A4 activity and sunitinib exposure using $7.5 \mathrm{mg}$ midazolam orally as a phenotypic probe.

Patient and methods: Serial blood samples for PK analysis of midazolam, 1-hydroxy-midazolam, and sunitinib were collected at steady-state sunitinib PK (between days 14 and 20). To confirm this observation in the mitotane-exposed patients, midazolam PK was evaluated in two additional patients with ACC and mitotane treatment.

Results: The four mitotane-treated patients showed highly induced CYP3A4 activity, even after interrupting mitotane therapy months before study entry, reflected by decreased midazolam exposure compared with the other seven patients (mean $\mathrm{AUC}_{0-12} \mathrm{~h}(95 \% \mathrm{CI}$ ): 7.6 (5.5-9.7) vs 139.0 (95.1-182.9) $\mu \mathrm{g} \times \mathrm{h} / \mathrm{l}$ respectively $P=0.001$ ) and increased 1-hydroxy-midazolam exposure (mean $\mathrm{AUC}_{0-12 \mathrm{~h}}(95 \% \mathrm{CI}): 409.6(290.5-528.7)$ vs 35.0 (26.4-43.6) $\left.\mu \mathrm{g} \times \mathrm{h} / \mathrm{l}, P=0.008\right)$. Sunitinib exposure was decreased in the two patients who were co-treated with mitotane $(267$ and $268 \mu \mathrm{g} \times \mathrm{h} / \mathrm{l}$ versus 1344 (1079-1609) (mean (95\% CI)) $\mu \mathrm{g} \times \mathrm{h} / \mathrm{l})$.

Conclusion: Mitotane has a strong and long-lasting inducing effect on CYP3A4 activity, which will result in clinically relevant interactions with multiple drugs since many drugs are metabolized by this enzyme.
\end{abstract}

European Journal of Endocrinology $164621-626$

\section{Introduction}

Mitotane (o,p'-DDD, $\mathrm{C}_{14} \mathrm{H}_{10} \mathrm{Cl}_{4}$, molecular weight 320.05 , poorly water soluble $(0.1 \mathrm{mg} / \mathrm{l}))$ is used to treat patients with adrenocortical carcinoma (ACC) $(1,2)$. The adrenocorticolytic effect of mitotane is generated by the covalent binding of reactive metabolic intermediates (acyl chloride metabolites) to still unknown adrenal proteins (3-6). Careful monitoring of serum mitotane levels is important, because mitotane has a narrow therapeutic window. Mitotane levels $>14 \mathrm{mg} / \mathrm{l}$ are required for the therapeutic effects, whereas serum drug levels $>20 \mathrm{mg} / \mathrm{l}$ correlate with considerable side effects, especially neurologic toxicity (7). As mitotane accumulates in adipose tissue, the plasma elimination half-life is extremely long (18-159 days). Consequently, it can take months to reach steady-state PK and, conversely, it also takes months to observe a decrease in plasma levels after discontinuation of mitotane (7). Unfortunately, many patients show progressive disease despite treatment with mitotane. Therefore, more effective additional treatment modalities are warranted, including polychemotherapy.

Surprisingly, there is hardly any information available on the metabolic pathways of mitotane, nor on the potential influence of mitotane on the metabolism of co-administered drugs. However, organochlorine insecticides, to which mitotane is chemically closely related, induce microsomal liver enzymes (8). In accordance, a case report described an interaction between mitotane and the anticoagulant warfarin which resulted in increased warfarin requirements, suggesting induction of metabolizing enzymes by mitotane (9). Additionally, a substantial increase in steroid requirement was 
described in two patients, but observed generally in clinical practice, on mitotane therapy which was explained by hepatic microsomal enzyme induction (10). Also an increase in bleeding time due to mitotane treatment was noticeably caused by defective platelet function (11). Controversially, mitotane-induced hypercholesterolemia as a result of increased cholesterol synthesis was believed to be caused by inhibition of cytochrome P450-mediated reactions (12).

In the present report, the effect of mitotane on cytochrome $\mathrm{P} 450$ (CYP) 3A4 activity is described using the phenotypic probe midazolam (13). Midazolam is extensively metabolized by CYP3A4 and, to a lesser extent, by CYP3A5 (14). It is used as a phenotypic probe to determine the activity of CYP3A4 (14). In addition, we describe the effect of mitotane on the exposure to an oral, multi-targeted tyrosine kinase inhibitor; sunitinib. Sunitinib is also metabolized by CYP3A4 to an equally active metabolite SU12662, which is further metabolized to inactive moieties by CYP3A4 (15).

\section{Patients and methods}

\section{Patients}

A remarkable effect on the PK of sunitinib and midazolam was observed in two patients with ACC who were exposed to mitotane. The original sunitinib PK study was designed to determine i) the relationship between CYP3A4 activity and sunitinib exposure and ii) the effect of grapefruit juice on sunitinib exposure (16). Both ACC patients showed an aberrant PK profile, e.g. highly reduced sunitinib and midazolam exposure and elevated 1-hydroxy-midazolam exposure. In order to confirm this serendipitous observation, the CYP3A4 activity in two additional patients on mitotane treatment was evaluated using midazolam as a phenotypic probe. Nine patients in total were included so far in the original PK study (16). Two patients (one male; 46 years old, $72 \mathrm{~kg}$, Eastern Cooperative Oncology Group (ECOG) performance status $=1$ and one female; 42 years old, $65 \mathrm{~kg}$, ECOG performance status=1) with metastatic ACC showed progressive disease despite mitotane therapy and were co-treated consecutively with sunitinib as an experimental therapy. The other seven patients (one female and six males; two gastrointestinal stromal tumors, two metastatic renal cell carcinoma, one prostate carcinoma, one chordoma, and one osteosarcoma; median (range) age $=60$ (41-77); weight $=82 \mathrm{~kg}$ (68-98); ECOG performance status $=1$ (0-1)) used sunitinib without mitotane therapy. The two additional patients (two females, 71-72 years old, 52-88 kg, ECOG performance status 1-1) were diagnosed with metastatic ACC and were treated with mitotane.

The initial study was approved by the institutional ethics committee (Leiden University Medical Center, The Netherlands), and the nine patients included in the original study patients gave written informed consent before entering the study. The inclusion of two additional patients was informally discussed with the institutional ethics committee, and they agreed with the rechallenge without a new study protocol since the outcome would contribute significantly to regular patient care in mitotane-treated patients. The inclusion of the two additional patients was therefore regarded as a diagnostic tool in regular patient care instead of protocol clinical study.

\section{Study design}

All patients in the initial PK study were treated with sunitinib $37.5-50 \mathrm{mg}$ once daily in a ' 4 weeks on -2 weeks off' dosing schedule. PK assessment of midazolam and sunitinib at steady-state sunitinib PK was performed between days 14 and 20. A single dose of midazolam $7.5 \mathrm{mg}$ was administered orally concomitantly with the regular dose of sunitinib. Blood samples were collected pre-dose, and 10, 20, 40 min; 1, 2, 3, 4, $5,6,7,8,10,12$, and $24 \mathrm{~h}$ after midazolam and sunitinib administration. The two patients who were included for confirmation of the serendipitous observation received only a single dose of midazolam $7.5 \mathrm{mg}$. Sunitinib was not co-administered to these two patients since they had no progressive disease under mitotane treatment. Blood samples in these patients were collected pre-dose, and 10, 20, $40 \mathrm{~min} ; 1,2,3,4,5$, $6,7,8,10$, and $12 \mathrm{~h}$ after midazolam administration.

\section{Measurement}

The mitotane concentrations in the four patients were measured by gas chromatographic electron capture detection assay (17). Sunitinib concentrations were quantified by liquid chromatographic-tandem mass spectrometric (LC-MS/MS) assay (18). Midazolam and 1-hydroxy-midazolam were measured using a validated LC-MS/MS assay. Briefly, $200 \mu \mathrm{l}$ plasma was extracted by adding $500 \mu \mathrm{l}$ of acetonitrile containing midazolam D4 $(4 \mu \mathrm{g} / \mathrm{l})$ and 1-hydroxymidazolam D4 (2 $\mu \mathrm{g} / \mathrm{l})$ as the internal standards, followed by vortex mixing and centrifugation at $38500 \boldsymbol{g}$ for $5 \mathrm{~min}$ at ambient temperature. The supernatant was collected, and $10 \mu \mathrm{l}$ was separated on an Atlantis T3 C18 analytical column $(2.1 \times 50 \mathrm{~mm}$, particle size $3 \mu \mathrm{m})$ and eluted with the following gradient (flow rate $(\mathrm{ml} / \mathrm{min}) /$ time $(\mathrm{min}) /$ percentage of solvent A (formic acid $0.1 \%$ in water)/ percentage of solvent $\mathrm{B}$ (formic acid $0.1 \%$ in acetonitrile)): $0.3 / 0.5 / 85 / 15 /, 0.3 / 1 / 10 / 90,0.3 / 4.3 / 10 / 90$, $0.5 / 0.01 / 10 / 90,0.5 / 0.39 / 85 / 15,0.5 / 3.3 / 85 / 15$, $0.3 / 0.05 / 85 / 15,0.3 / 0.05 / 85 / 15$. The effluent was monitored with a Micromass Quattro LC triplequadrupole mass-spectrometric detector (Waters, Milford, MA, USA) using the electrospray positive ionization mode. The calibration lines of midazolam and 1-hydroxy-midazolam were linear over the range of 
Table 1 Patient characteristics.

\begin{tabular}{|c|c|c|}
\hline \multirow[b]{2}{*}{ Characteristics } & \multicolumn{2}{|c|}{ Value } \\
\hline & Non-mitotane users & Mitotane users \\
\hline Number of patients & 7 & 4 \\
\hline Sex (female/male) & $1 / 6$ & $3 / 1$ \\
\hline $\mathrm{Age}^{\star}$ (years) & $62(40-77)$ & $58(44-72)$ \\
\hline \multicolumn{3}{|c|}{ Baseline renal and liver function parameters } \\
\hline Creatinine $^{*}(\mu \mathrm{M})$ & $85(56-122)$ & $69(56-77)$ \\
\hline Total bilirubin* $(\mu \mathrm{M})$ & $9(6-15)$ & $6(3-9)$ \\
\hline $\mathrm{ALT}^{*}$ (units/l) & $29(14-47)$ & $36(12-68)$ \\
\hline \multicolumn{3}{|c|}{ Baseline hematologic function parameters } \\
\hline $\mathrm{Hb}^{*}(\mathrm{mM})$ & $8.5(7.4-9.4)$ & $7.5(6.9-8.7)$ \\
\hline $\mathrm{WBC}^{*}\left(\times 10^{9} / \mathrm{I}\right)$ & $5.0(3.5-6.8)$ & $16.4(3.6-38.2)$ \\
\hline Thrombocytes* $\left(\times 10^{9} / \mathrm{l}\right)$ & $182(122-263)$ & $357(198-490)$ \\
\hline
\end{tabular}

${ }^{*}$ Mean with ranges.

$1-100$ and $0.5-50 \mathrm{ng} / \mathrm{ml}$ respectively. The within-day and between-day precision and accuracy were $<5 \%$. The LLQ of the midazolam and 1-hydroxy-midazolam assay were both $0.3 \mathrm{ng} / \mathrm{ml}$.

\section{Pharmacokinetic analysis}

Sunitinib, midazolam, and 1-hydroxy-midazolam plasma exposure was assessed by non-compartmental methods using WinNonlin (version 5.2.1; Pharsight Corporation, Mountain View, CA, USA). Midazolam and 1-hydroxy-midazolam exposure $\left(\mathrm{AUC}_{0-12 \mathrm{~h}}\right)$ was assessed over $12 \mathrm{~h}$ since midazolam and 1-hydroxymidazolam have a short half-life (1.0-3.5 h and $0.8-1.0 \mathrm{~h}$ respectively), and therefore the elimination was nearly completed at $12 \mathrm{~h}$ post-dose. Sunitinib exposure $\left(\mathrm{AUC}_{0-24 \mathrm{~h}}\right)$ was assessed over $24 \mathrm{~h}$. Since two patients received $37.5 \mathrm{mg}$ sunitinib, the calculated AUC was normalized to a sunitinib dose of $50 \mathrm{mg}$ (=DAUC). The mitotane concentrations of the mitotane users were determined in the pre-dose blood sample.

\section{Statistical analysis}

Statistical analysis was performed with an independent sample $t$-test in which the area under the concentration time curves of midazolam and 1-hydroxy-midazolam in mitotane users were compared with non-mitotaneexposed patients. $P$ values $<0.05$ were considered to be statistically significant. Calculations were done using SPSS version 16.0 software, (SPSS, Chicago, IL, USA).

\section{Results}

\section{Clinical characteristics of patients with ACC}

Patient 1, a 44-year-old man, was diagnosed in March 2007 with ACC in his right adrenal gland of 3.5 inch. The primary tumor was extirpated. However, in May 2007, there was local and distal recurrence of ACC for which mitotane therapy was started $3.5 \mathrm{~g}$ tid. Because standard systemic anti-tumor therapies had failed, sunitinib was prescribed as an experimental therapy in December 2007 in addition to the mitotane therapy. In January 2008, the patient volunteered for the pharmacokinetic study.

Patient 2, a 44-year-old woman, was diagnosed in June 2005 with ACC in her right adrenal gland with two hepatic metastases. Mitotane therapy started in June 2005 and continued until August 2007. After failure of standard systemic anti-tumor therapies, she was started on sunitinib as an experimental therapy in October 2007. In October 2007, the patient volunteered to participate in the pharmacokinetic study.

Patient 3, a 71-year-old woman, was diagnosed in September 2006 with ACC in her right adrenal gland. Mitotane treatment started (500 mg tid) at the end of 2006 after metastatic disease was diagnosed and continues at the time of writing this paper (August 2010). In March 2010, the patient volunteered to participate in the midazolam pharmacokinetic study.
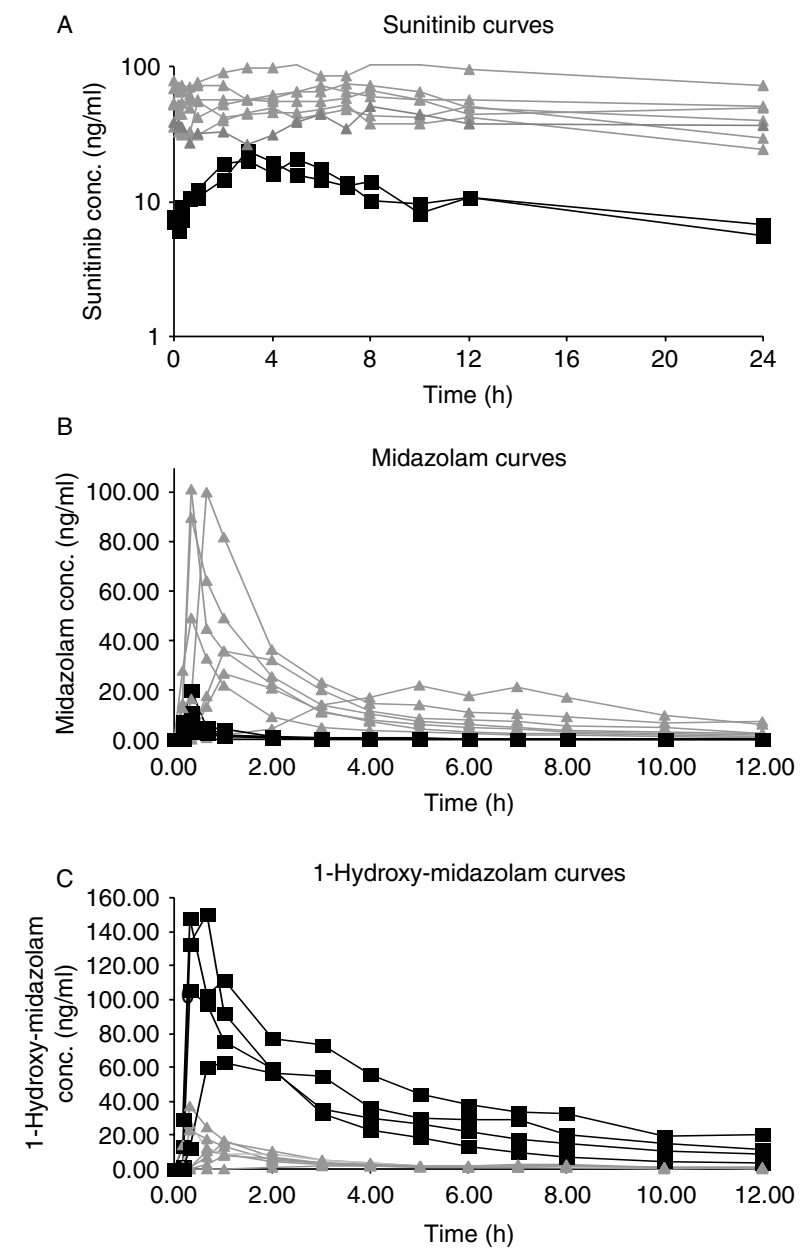

Figure 1 Individual plasma concentrations of (A) sunitinib, (B) midazolam, and (C) 1-hydroxy-midazolam. The two black sunitinib curves and four black midazolam and 1-hydroxy-midazolam curves represent the mitotane-treated patients; the gray curves represent the seven non-mitotane-treated patients. 
Table 2 Mitotane, midazolam, 1-hydroxy-midazolam, and sunitinib exposure. Mean values are shown with 95\% confidence interval in parentheses $(95 \% \mathrm{Cl})$.

\begin{tabular}{|c|c|c|c|c|c|c|}
\hline \multirow[b]{2}{*}{ Parameters } & \multicolumn{5}{|c|}{ Mitotane-treated patients $(n=4)$} & \multirow[b]{2}{*}{ Other patients $(n=7)$} \\
\hline & Patient 1 & Patient 2 & Patient 3 & Patient 4 & Mean $(95 \% \mathrm{Cl})$ & \\
\hline Mitotane daily dose (g) & $3 \times 3.5$ & $3 \times 1.0$ & $3 \times 0.5$ & $\begin{array}{l}1 \times 0.5 \text { e.o.d. } \\
2 \times 0.5 \text { e.o.d }\end{array}$ & & NA \\
\hline Status mitotane treatment & Ongoing & Stopped $^{\dagger}$ & Ongoing & Ongoing & & NA \\
\hline Mitotane concentration (mg/l) & 8.1 & 4.9 & 17.8 & 9.0 & & NA \\
\hline Midazolam $\mathrm{AUC}_{0-12 \mathrm{~h}}(\mu \mathrm{g} \times \mathrm{h} / \mathrm{l})$ & 6.5 & 10.1 & 8.6 & 5.3 & $7.6(5.5-9.7)$ & $139.0(95.1-182.9)$ \\
\hline $1-\mathrm{OH}$ midazolam $\mathrm{AUC} \mathrm{C}_{0-12 \mathrm{~h}}(\mu \mathrm{g} \times \mathrm{h} / \mathrm{l})$ & 292.3 & 390.7 & 375.4 & 580.0 & $409.6(290.5-528.7)$ & $35.0(26.4-43.6)$ \\
\hline Sunitinib DAUC $\mathrm{D}_{0-24 \mathrm{~h}}(\mu \mathrm{g} \times \mathrm{h} / \mathrm{l})$ & 268 & 267 & NA & NA & & $1344(1079-1609)$ \\
\hline
\end{tabular}

e.o.d., every other day; $\mathrm{m}$, months; $\mathrm{AUC}_{0-12 \mathrm{~h}}$, area under the plasma concentration time curve over the observed interval; $\mathrm{DAUC} \mathrm{C}_{0-24 \mathrm{~h}}$, dose-normalized (to $50 \mathrm{mg}$ sunitinib) area under the plasma concentration time curve.

${ }^{\dagger}$, stopped 2.5 months before study entry.

Patient 4, a 72-year-old woman, was diagnosed in April 2006 with ACC in her left adrenal gland. In August 2008, there was a local recurrence of ACC. Mitotane treatment was started in September 2008 (500 mg oid e.o.d. and $500 \mathrm{mg}$ bid e.o.d.), and the treatment was continued at the moment of this report (August 2010). In April 2010, the patient volunteered to participate in the midazolam pharmacokinetic study. Other patient characteristics of mitotane and nonmitotane-treated patients are summarized in Table 1.

\section{Pharmacokinetic data}

Mitotane has an extremely long elimination half-life (18-159 days) and, consequently, an effect of mitotane on co-administered drugs is still present although mitotane therapy had been discontinued several months previously (patient 2) (19). Indeed, the mitotane serum concentrations were 8.1, 4.9, 17.8, and $9.0 \mathrm{mg} / \mathrm{l}$ in patients $1-4$ respectively. The mitotaneexposed patients showed highly induced CYP3A4 activity reflected in decreased sunitinib and midazolam exposure (including increased 1-hydroxy-midazolam exposure) (Fig. 1; Table 2). The two mitotane-treated patients showed markedly reduced sunitinib exposure $\left(\right.$ DAUC $_{0-24 \mathrm{~h}}$ ) compared to the other seven patients (267 and $268 \mu \mathrm{g} \times \mathrm{h} / \mathrm{l}$ versus 1344 (1079-1609; mean $(95 \%$ CI $) \mu \mathrm{g} \times \mathrm{h} / \mathrm{l}$ respectively, Fig. $1 \mathrm{~A})$ as well as compared with sunitinib exposure levels reported in literature (mean $\mathrm{AUC}_{0-24 \mathrm{~h}}: 965 \mu \mathrm{g} \times \mathrm{h} / \mathrm{l}$ (20) and $1296 \mu \mathrm{g} \times \mathrm{h} / \mathrm{l}(21))$.

In addition, mitotane treatment was associated with strikingly reduced midazolam exposure $\left(\mathrm{AUC}_{0-12 \mathrm{~h}}\right)$ in all four patients compared to the midazolam exposure measured in the other patients (mean $\mathrm{AUC}_{0-12 \mathrm{~h}}(95 \% \mathrm{CI})$ : $7.6(5.5-9.7)$ vs $139.0(95.1-182.9) \mu \mathrm{g} \times \mathrm{h} / \mathrm{l}$ respectively, $P=0.001$, Fig. 1B). Examples of dose-normalized $(7.5 \mathrm{mg})$ midazolam exposure levels reported in literature are: $\mathrm{AUC}_{0-12 \mathrm{~h}}: 116 \mu \mathrm{g} \times \mathrm{h} / \mathrm{l}$ (22) and $\mathrm{AUC}_{0-\infty}$ $120.6 \mu \mathrm{g} \times \mathrm{h} / \mathrm{l}$ (23). Midazolam is metabolized by CYP3A4 into 1-hydroxy-midazolam and to a lesser extent into 4-hydroxy-midazolam. Indeed, the four patients treated with mitotane showed highly elevated 1-hydroxy-midazolam exposure levels $\left(\mathrm{AUC}_{\mathrm{O}-12 \mathrm{~h}}\right)$ compared to the other patients (mean $\mathrm{AUC}_{0-12 \mathrm{~h}}$ $(95 \% \mathrm{CI}):=409.6(290.5-528.7) \mu \mathrm{g} \times \mathrm{h} / \mathrm{l}$ versus 35.0 (26.4-43.6) $\mu \mathrm{g} \times \mathrm{h} / \mathrm{l}$ respectively, $P=0.008$, Fig. $1 \mathrm{C}$ ), indicative of increased CYP3A4 activity.

\section{Discussion}

Mitotane treatment was associated with induced metabolism of midazolam as well as of sunitinib in the two patients in the original PK study. This serendipitous observation was confirmed in two additional patients, who were treated with mitotane and also showed markedly induced midazolam metabolism. Since midazolam is mainly metabolized by CYP3A4 with little affinity for CYP3A5, ABCB1, and ABCG2, our observation is most likely the result of a strong inducing effect of mitotane on CYP3A4 activity $(14,24,25)$. The small number of patients in this report may seem as a limitation. However, the effect of mitotane on CYP3A4 activity is very convincing since the exposure to the parent compound (midazolam) as well as the metabolite (1-hydroxy-midazolam) shows a similar pattern in each patient. Additionally, the levels of midazolam and 1-hydroxy-midazolam in the rechallenged patients were exactly as expected.

This observation is clinically highly relevant, since many drugs are metabolized through CYP3A4, e.g. simvastatin, clarithromycin, cyclosporine (26). Consequently, co-administration of mitotane will result in considerable drug-drug interactions, as observed with midazolam and sunitinib. This inducing effect of mitotane on CYP3A4 is apparently extremely potent, even in comparison with the strongest CYP3A4 inducer described yet, rifampicin (27).

Three out of the four patients had a subtherapeutic mitotane plasma concentration at the moment of the drug interaction study (i.e. $<14 \mathrm{mg} / \mathrm{l}$ ). The mitotane 
exposure levels might be of influence on the magnitude of the effect on CYP3A4. However, there was no relationship observed between mitotane exposure levels and the reduced midazolam exposure and increased 1-hydroxymidazolam exposure in this small group of patients.

In conclusion, in this pharmacokinetic study, we observed a very strong CYP3A4-inducing effect of mitotane, which led to a considerable drug-drug interaction with sunitinib, even after 2 months of cessation of mitotane therapy. This strong CYP3A4-inducing effect of mitotane will also affect the PK of other drugs, which are metabolized by CYP3A4 and thus can result in considerable drug-drug interactions. We cannot exclude additional effects of mitotane on other metabolizing enzymes. Therefore, all physicians treating ACC patients with mitotane should be aware of these potential drug interactions, which can result in inadvertent therapeutic failure of the co-administered drugs.

\section{Declaration of interest}

The authors declare that there is no conflict of interest that could be perceived as prejudicing the impartiality of the research reported.

\section{Funding}

This research did not receive any specific grant from any funding agency in the public, commercial, or not-for-profit sector.

\section{Acknowledgements}

We thank J Ouwerkerk and M A G den Hollander for their help with data and sample collection, J M C König-Quartel for her help with data analysis and A Sparreboom and S Baker for the scientific contribution.

\section{References}

1 Bergenstal DM, Lipsett MB, Moy RH \& Hertz R. Regression of adrenal cancer and suppression of adrenal function in man by o,p DDD. Transactions of the Association of American Physicians 195972 341-350.

2 Allolio B, Hahner S, Weismann D \& Fassnacht M. Management of adrenocortical carcinoma. Clinical Endocrinology $2004 \mathbf{6 0}$ 273-287. (doi:10.1046/j.1365-2265.2003.01881.x)

3 Cai W, Counsell RE, Schteingart DE, Sinsheimer JE, Vaz AD \& Wotring LL. Adrenal proteins bound by a reactive intermediate of mitotane. Cancer Chemotherapy and Pharmacology 199739 537-540. (doi:10.1007/s002800050610)

4 Cai W, Counsell RE, Djanegara T, Schteingart DE, Sinsheimer JE \& Wotring LL. Metabolic activation and binding of mitotane in adrenal cortex homogenates. Journal of Pharmaceutical Sciences 199584 134-138. (doi:10.1002/jps.2600840203)

5 Martz F \& Straw JA. Metabolism and covalent binding of 1-(o-chlorophenyl)-1-(p-chlorophenyl)-2,2-dichloroethane (o,p,'-DDD). Correlation between adrenocorticolytic activity and metabolic activation by adrenocortical mitochondria. Drug Metabolism and Disposition 1980 8 127-130.

6 Hahner S \& Fassnacht M. Mitotane for adrenocortical carcinoma treatment. Current Opinion in Investigational Drugs 20056 386-394.

7 Baudin E, Pellegriti G, Bonnay M, Penfornis A, Laplanche A, Vassal G \& Schlumberger M. Impact of monitoring plasma 1,1-dichlorodiphenildichloroethane (o,p'DDD) levels on the treatment of patients with adrenocortical carcinoma. Cancer 200192 1385-1392. (doi:10.1002/1097-0142(20010915) 92:6<1385::AID-CNCR1461>3.0.CO;2-2)

8 Street JC. VI. Biochemical and pathological effects. Organochlorine insecticides and the stimulation of liver microsome enzymes. Annals of the New York Academy of Sciences $1969160274-290$. (doi:10.1111/j.1749-6632.1969.tb15848.x)

9 Cuddy PG \& Loftus LS. Influence of mitotane on the hypoprothrombinemic effect of warfarin. Southern Medical Journal $1986 \mathbf{7 9}$ 387-388. (doi:10.1097/00007611-198603000-00037)

10 Hague RV, May W \& Cullen DR. Hepatic microsomal enzyme induction and adrenal crisis due to o,p'DDD therapy for metastatic adrenocortical carcinoma. Clinical Endocrinology 198931 51-57. (doi:10.1111/j.1365-2265.1989.tb00453.x)

11 Haak HR, Caekebeke-Peerlinck KM, van Seters AP \& Briet E. Prolonged bleeding time due to mitotane therapy. European Journal of Cancer 199127 638-641. (doi:10.1016/0277-5379(91)90233-4)

12 Maher VM, Trainer PJ, Scoppola A, Anderson JV, Thompson GR \& Besser GM. Possible mechanism and treatment of o,p'DDD-induced hypercholesterolaemia. Quarterly Journal of Medicine $1992 \mathbf{8 4}$ 671-679.

13 Magnusson MO, Dahl ML, Cederberg J, Karlsson MO \& Sandstrom R. Pharmacodynamics of carbamazepine-mediated induction of CYP3A4, CYP1A2, and Pgp as assessed by probe substrates midazolam, caffeine, and digoxin. Clinical Pharmacology and Therapeutics $2008 \mathbf{8 4} 52-62$. (doi:10.1038/sj.clpt.6100431)

14 Fuhr U, Jetter A \& Kirchheiner J. Appropriate phenotyping procedures for drug metabolizing enzymes and transporters in humans and their simultaneous use in the "cocktail" approach. Clinical Pharmacology and Therapeutics 200781 270-283. (doi:10. 1038/sj.clpt.6100050)

15 Goodman VL, Rock EP, Dagher R, Ramchandani RP, Abraham S, Gobburu JV, Booth BP, Verbois SL, Morse DE, Liang CY, Chidambaram N, Jiang JX, Tang S, Mahjoob K, Justice R \& Pazdur R. Approval summary: sunitinib for the treatment of imatinib refractory or intolerant gastrointestinal stromal tumors and advanced renal cell carcinoma. Clinical Cancer Research 2007 13 1367-1373. (doi:10.1158/1078-0432.CCR-06-2328)

16 van Erp NP, Baker SD, Zandvliet AS, Ploeger BA, den HM, Chen Z, den HJ, Konig-Quartel JM, Guchelaar HJ \& Gelderblom H. Marginal increase of sunitinib exposure by grapefruit juice. Cancer Chemotherapy and Pharmacology, 2010 In press. (doi:10.1007/s00280-010-1367-0)

17 Moolenaar AJ, Niewint JW \& Oei IT. Estimation of o,p'-DDD in plasma by gas-liquid chromatography. Clinica Chimica Acta 1977 76 213-218. (doi:10.1016/0009-8981(77)90098-5)

18 Minkin P, Zhao M, Chen Z, Ouwerkerk J, Gelderblom H \& Baker SD. Quantification of sunitinib in human plasma by high-performance liquid chromatography-tandem mass spectrometry. Journal of Chromatography. B, Analytical Technologies in the Biomedical and Life Sciences 2008 874 84-88. (doi:10.1016/j.jchromb.2008.09.007)

19 Bristol-Myers Squibb. Drug label Lysodren approved 28/04/2004. Available at: http://www.emea.europa.eu/, Accessed on August 2010.

20 Houk BE, Bello CL, Kang D \& Amantea M. A population pharmacokinetic meta-analysis of sunitinib malate (SU11248) and its primary metabolite (SU12662) in healthy volunteers and oncology patients. Clinical Cancer Research 200915 2497-2506. (doi:10.1158/1078-0432.CCR-08-1893)

21 Faivre S, Delbaldo C, Vera K, Robert C, Lozahic S, Lassau N, Bello C, Deprimo S, Brega N, Massimini G, Armand JP, Scigalla P \& Raymond E. Safety, pharmacokinetic, and antitumor activity of SU11248, a novel oral multitarget tyrosine kinase inhibitor, in patients with cancer. Journal of Clinical Oncology 200624 25-35. (doi:10.1200/JC0.2005.02.2194)

22 Mueller SC, Majcher-Peszynska J, Mundkowski RG, Uehleke B, Klammt S, Sievers H, Lehnfeld R, Frank B, Thurow K, Kundt G \& Drewelow B. No clinically relevant CYP3A induction after St. John's wort with low hyperforin content in healthy volunteers. European Journal of Clinical Pharmacology 200965 81-87. (doi:10. 1007/s00228-008-0554-y)

23 Farkas D, Oleson LE, Zhao Y, Harmatz JS, Zinny MA, Court MH \& Greenblatt DJ. Pomegranate juice does not impair clearance of oral or 
intravenous midazolam, a probe for cytochrome P450-3A activity: comparison with grapefruit juice. Journal of Clinical Pharmacology 200747 286-294. (doi:10.1177/0091270006298359)

24 Kim RB, Wandel C, Leake B, Cvetkovic M, Fromm MF, Dempsey PJ, Roden MM, Belas F, Chaudhary AK, Roden DM, Wood AJ \& Wilkinson GR. Interrelationship between substrates and inhibitors of human CYP3A and P-glycoprotein. Pharmaceutical Research 199916 408-414. (doi:10.1023/A:1018877803319)

25 Kurnik D, Wood AJ \& Wilkinson GR. The erythromycin breath test reflects P-glycoprotein function independently of cytochrome P450 3A activity. Clinical Pharmacology and Therapeutics 2006 80 228-234. (doi:10.1016/j.clpt.2006.06.002)
26 The Trustees of Indiana University. P450 Drug Interaction Table. Available at: http://medicine.iupui.edu/clinpharm/ddis/table.asp, accessed June 2009.

27 Chung E, Nafziger AN, Kazierad DJ \& Bertino JS Jr. Comparison of midazolam and simvastatin as cytochrome P450 3A probes. Clinical Pharmacology and Therapeutics 200679 350-361. (doi:10. 1016/j.clpt.2005.11.016)

Received 6 January 2011

Accepted 10 January 2011 